\title{
Implementing Multiagent Systems Technology for Power Distribution Network Control and Protection Management
}

\author{
Ioannis S. Baxevanos and Dimitris P. Labridis, Senior Member, IEEE
}

\begin{abstract}
Power distribution network protection and restoration are significant issues in terms of quality and reliability of the supplied electrical energy. In this paper, an extended research in possible high-end protection and control methods is presented. The authors intend to show the efficiency of combining modern IT techniques with the equipment provided by distribution automation evolving technology. As a result, a flexible and versatile multiagent system (MAS) is proposed. The effected MAS is fault isolation and power restoration oriented, as shown in the case studies. The structure of the proposed system (software and hardware aspects) is introduced. Both microscopic and macroscopic procedures are revised. Power line communications technology is implemented. Results acquired from computer simulations are evaluated. Conclusions concerning the perspectives of implementing the proposed system are finally presented.
\end{abstract}

Index Terms-Artificial intelligence, cooperative systems, power distribution protection, underground power distribution lines.

\section{INTRODUCTION}

D ISTRIBUTION system protection and restoration are important applications of the artificial intelligence technology. Researchers have already associated available knowledge and experience of domain experts with computational systems dedicated to centralized control and protection. As a result, several rule-based expert systems have been proposed including logical rules for switching actions for fault isolation and load transfer between adjacent feeders [1], heuristic rules (compiled from the dispatcher's experience) for locating distribution system faults [2] and to some extent, presenting user-friendly graphical user interfaces (GUIs) as practical restoration aid systems, to assist the decision making of supervisory-control and data-acquisition (SCADA) operators [3]. Expert systems have also been applied to the general concept of power system restoration as shown in [4]-[6]. Possessing an auxiliary role, expert systems usually require information concerning the topology of the network and the nature of the network's components, as well as remote metering of electrical values. Therefore dedicated communication channels are postulated, including fiber optics or copper-wire telecommunications and recently, wireless technologies such as Global System for Mobile Communications (GSM) and terrestrial

Manuscript received June 6, 2005; revised December 9, 2005. This work was supported by the Operational Program for Education and Initial Vocational Training (O.P. "Education")-Heracletus. Paper no. TPWRD-00334-2005.

The authors are with the Department of Electrical and Computer Engineering, Power Systems Laboratory, Aristotle University of Thessaloniki, Thessaloniki GR 54124, Greece (e-mail: baxi@auth.gr).

Digital Object Identifier 10.1109/TPWRD.2006.877085 trunked radio (TETRA) [7]. However, issues regarding time and computational resources, costs and physical aspects of the problems restrain the operability of the above systems.

Efforts toward distributing activities and intelligence fall into the scope of distributed AI (DAI) and multiagent systems (MAS). Implementing the agent technology and especially MAS for infrastructures, such as the power grid, seems to be quite promising [8], [9]. In the case of distribution system fault management, the decomposition of the problem is quite effective [10]. Approaching the complex problem of distribution grid management in a distributed way converges with significant areas of applications for MAS, namely distributed solving of problems and solving distributed problems [11].

The utility industries will benefit from the implementation of multiagent technology, because there will be no need for exclusive central control and protection of the distribution grid. Supervision of procedures that will take place locally, as close as possible to the problematic sectors, will only be needed. Several heterogeneous components with possible incompatible behaviors will combine actions under the guidance of software agents that will cooperate in order to accomplish their joint goals.

The objectives of the MAS approach to distribution network management are still the same with the ones of centralized systems: to minimize the duration of outages as well as the customer interruptions, in favor of the overall distribution system reliability. Customer-based distribution reliability indices, especially customer average interruption duration index (CAIDI) and system average interruption duration index (SAIDI) [12], will be potentially improved. However, DAI and MAS technology, if implemented, may actually result in self-managing automated networks [13].

The MAS project presented in this paper intends to reinforce self-managing capabilities provided to the network, with adaptive behaviors which are necessary for applications in many different network topologies.

\section{PRESEnt Work ApPlication Field}

This particular research and the effected project were triggered off by the imperative need for really rapid fault localization, isolation and restoration procedures. A brief layout of a distribution network that falls into the scope of this work is presented in Fig. 1 [14]. Open-loop structures as well as radial medium-voltage (MV) feeders are considered.

The distribution network consists of both underground (residential, commercial, and industrial customers) and overhead (industrial and agricultural customers) MV lines. Statistical data indicate that underground lines usually sustain permanent 


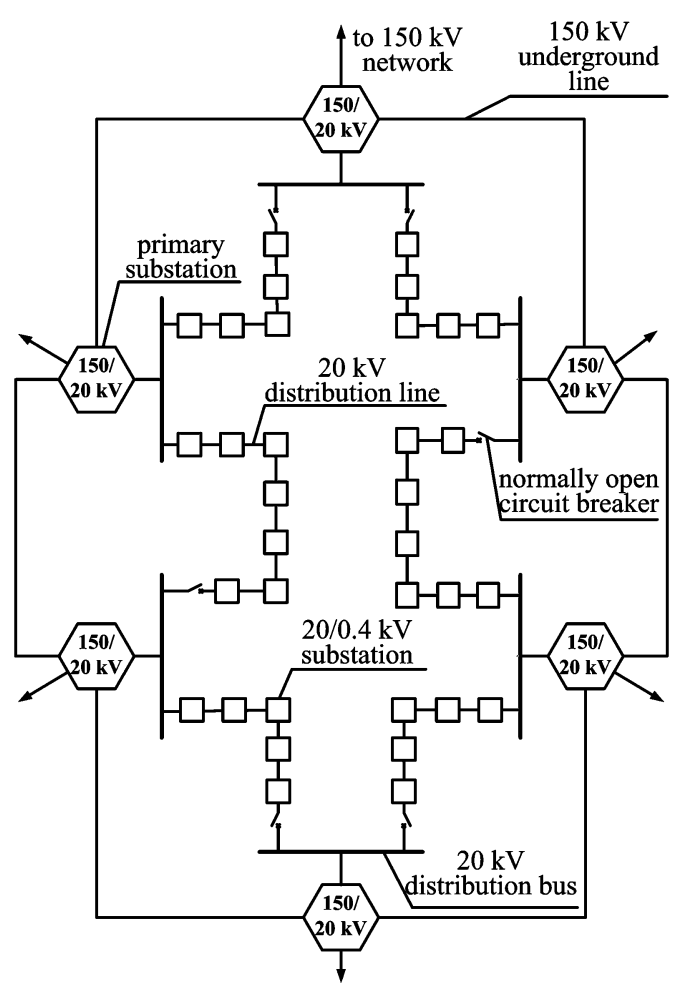

Fig. 1. Topological layout of a typical power distribution network.

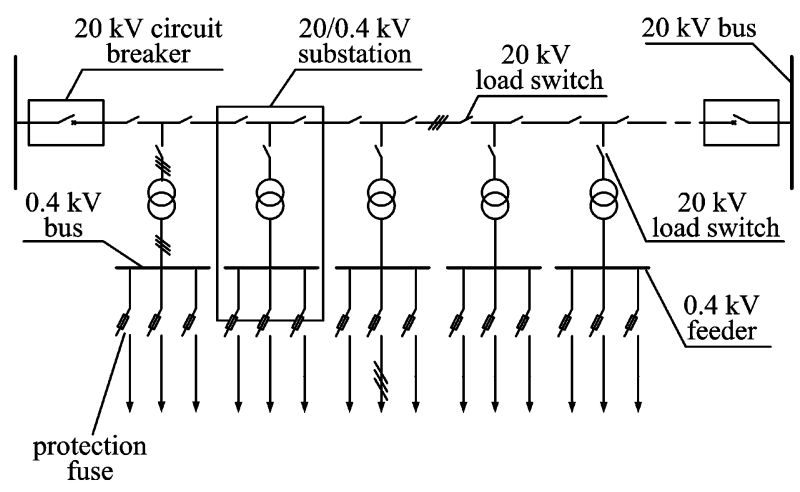

Fig. 2. Underground MV line (rated at $20 \mathrm{kV}$ ).

outages while overhead lines usually undergo temporary ones. Therefore, the protection systems vary according to the types of faults considered (permanent or temporary). This work focuses on the underground lines, since locating a possible underground line fault, after the fault has occurred and been cleared, is a difficult task to carry out. Practical issues concerning the inspection of underground infrastructures as well as technical constraints regarding the centralized acquisition of multiple fault detection readings from network nodes, increase the difficulty level. Moreover, fast and accurate fault isolation and power restoration procedures are tasks difficult to accomplish, especially in underground distribution networks with a lot of interconnections and different power supplies. A typical but realistic underground MV line, equipped with protective and switching devices, is shown in Fig. 2.

Control and subcontrol centers are sited at the $150 / 20-\mathrm{kV}$ substations. Data concerning the MV line currents, voltages, and apparent power are available from SCADA systems. The state of the $20-\mathrm{kV}$ breakers is controlled locally or/and remotely via dedicated communication copper lines and modems.

Since it is currently unattainable to automate the cable repairing procedure, the authors propose techniques aimed at minimizing the time requirements of the fault localization, isolation, and power restoration of the healthy network sectors.

It should be noted that this research considers technically advanced distribution networks by means of the following assumptions.

1) All of the $20-\mathrm{kV}$ load switches, located at the $20 / 0.4-\mathrm{kV}$ substations, are equipped with motorized mechanisms controlled by switching relays.

2) Intelligent electronic devices (IEDs) for data acquisition (plus fault detection readings) and operating command dispatching are also utilized. The IEDs' role is restricted to record the electrical parameters of the substation and to indicate local fault detection.

3) Inductive and/or capacitive couplers along with MV modems will enable power-line communications (PLC). Phase-to-phase or phase-to-ground coupling schemes may be implemented.

4) Low-performance computers with high protection standards, powered by uninterruptible power supplies (UPS) are installed at the 20/0.4-kV substations. The distributed MAS is accommodated in those computers.

\section{Structure of the Multiagent System}

The proposed architecture presents a dual-level structure. The first level considers the implementation of similar groups of collaborating software agents (agent communities) located at significant network nodes which are the 20/0.4-kV distribution substations. The second level concerns the formation of the above groups in the substation environment. Thus the fault isolation and power restoration problem is being distributed among the agent communities. In the substation environment, the necessary activities and actions are distributed among the members of the respective agent community.

In order to comply with the requirements of the MAS principles, the authors developed the agent communities with JADE [15], a MAS development framework which conforms to FIPA [16] standards for intelligent agents.

The design of the system resulted in three software agents to constitute each agent community:

Agent Expert. It is a software agent that has been assigned the task to handle emerging situations. Equipped with the appropriate knowledge representation, beliefs about its environment and explicit goals, this agent combines data, assesses each situation separately, and decides on the community's actions.

Agent Inter. It is a software entity responsible for providing Agent Expert all necessary connections with the physical environment. It possesses the appropriate knowledge to operate the programmable controllers and to decide on issues that affect the synchronization of the system.

Agent Com. An agent responsible for the communication issues among the agent communities. It initializes the communications between adjacent network nodes as well as between the substations and the Terminal Agents. 
Apart from the three-agent structured communities, which are located at the $20 / 0.4 \mathrm{kV}$ substations, individual agents are also introduced with explicit tasks:

- to operate the circuit breakers (CBs) according to the messages they receive from Agents Com;

- to inform the control engineers about the location of the isolated fault;

- to send informative messages to the agent communities, containing the state and load conditions of the CBs.

The specified location of those agents is the distribution line terminals (i.e., the 150/20-kV substations, therefore named Terminal Agents).

\section{AgEnt INTELLigenCE}

The three-agent schema entailed the minimum number of activities that have to be performed in a $20 / 0.4-\mathrm{kV}$ distribution substation, without causing any operational defects: communicating, switching device handling, and decision making. Special care was taken regarding the versatility and the consistency of the agent communities. Different agents perform different and clearly distinct tasks without any overlapping phenomena. Moreover, the degree of intelligence of the agents is specified, according to the tasks and activities that had been assigned to each of them separately. The community's agents comprise the rule-based expert systems and an agent software framework. The expert systems are programmed in PROgramming in LOGic (PROLOG) [17], which is a logic programming language allowing for the minimum semantic gap for knowledge representation. Along with a robust inference engine, the PROLOG logic base is encapsulated in the agent framework. In the following, the agent communities are referred as multiagent system communities (MASC).

\section{A. Prior Knowledge}

The distribution of the problem requires that the MASCs keep prior knowledge regarding local environments. The notion of prior knowledge is formulated and stored in lists of the form

$$
\begin{aligned}
\text { List_1 : } & {[\operatorname{switch}(X), \operatorname{load}(Y), \operatorname{state}(Z),} \\
& \text { fault_detect }(W), \operatorname{sub}(A), \operatorname{power}(B), \\
& \operatorname{fault}(C), \operatorname{breaker}(D), \text { b_load }(E), \\
& \operatorname{remain}(F), \operatorname{status}(G)] .
\end{aligned}
$$

The maximum number of lists is determined by the number of the MV load switches that an MASC controls. The elements of the lists are inter-related objects of the 20/0.4-kV substation environment and they are utilized by the agents as reference tables. The description of the list's elements in (1) is as follows

$$
\begin{array}{ll}
\operatorname{switch}(X) & \text { element that refers to an MV load } \\
& \text { switch. } X \text { is the MV load switch } \\
& \text { identifier with possible values } X=1, \\
& 2,3,4 ; \\
& \text { element that refers to the actual load } \\
& \text { in [kilovolt-amperes] of an MV load } \\
& \text { switch. } Y \text { is the load of switch with } \\
& \text { identifier } X . Y \text { is an integer; }
\end{array}
$$

$\operatorname{state}(Z)$

fault_detect $(W)$

$\operatorname{sub}(A)$

$$
\begin{aligned}
& \text { power }(B) \quad \text { element that refers to the load in } \\
& \text { (kilovolta-amperes) of a } 20 / 0.4-\mathrm{kV} \\
& \text { substation. } B \text { is the load of the } \\
& \text { substation with identifier } A . B \text { is an } \\
& \text { integer; } \\
& \text { fault }(C) \quad \text { element that refers to the status of fault } \\
& \text { detection of a } 20 / 0.4-\mathrm{kV} \text { substation. } \\
& C \text { is the status (i.e., } 1 / 0 \text { for yes/no) of } \\
& \text { the fault detection of substation } A \text {. } C \\
& \text { is a Boolean; } \\
& \text { breaker }(D) \quad \text { element that refers to a supplying } \\
& \text { breaker. } D \text { is the string identifier of a } \\
& \text { supplying breaker; } \\
& \text { b_load }(E) \quad \text { element that refers to the load in } \\
& \text { (kilovolt-amperes) of a supplying } \\
& \text { breaker. } E \text { is the load of a breaker } \\
& \text { with identifier } D \text {. } E \text { is an integer; } \\
& \operatorname{remain}(F) \quad \text { element that refers to the remaining } \\
& \text { load (in kilovolt amperes) of a } \\
& \text { supplying breaker. } F \text { is the remaining } \\
& \text { load that breaker with identifier } D \text { can } \\
& \text { serve. } F \text { is an integer; } \\
& \operatorname{status}(G) \quad \text { element that refers to the state of } \\
& \text { a supplying breaker. } G \text { is the state } \\
& \text { (i.e., 1/0 for on/off) of breaker with } \\
& \text { identifier D. } G \text { is a Boolean. }
\end{aligned}
$$

An MASC updates the values of the lists' elements according to readings from the IEDs and incoming messages from adjacent MASCs. For example, if the sender of an incoming message is $\operatorname{sub}(A)$, then the receiver may easily conclude that this message is coming from the side of $\operatorname{switch}(X)$. The receiver will make use of this piece of information for further reasoning.

\section{B. Operational States}

The MASCs exhibit three discrete operational states.

1) steady state;

2) fault isolation state;

3) restoration state.

The MASCs change operational states depending on the environmental conditions. On steady-state, Agent Expert controls the operation of the community while Agents Com and Inter 


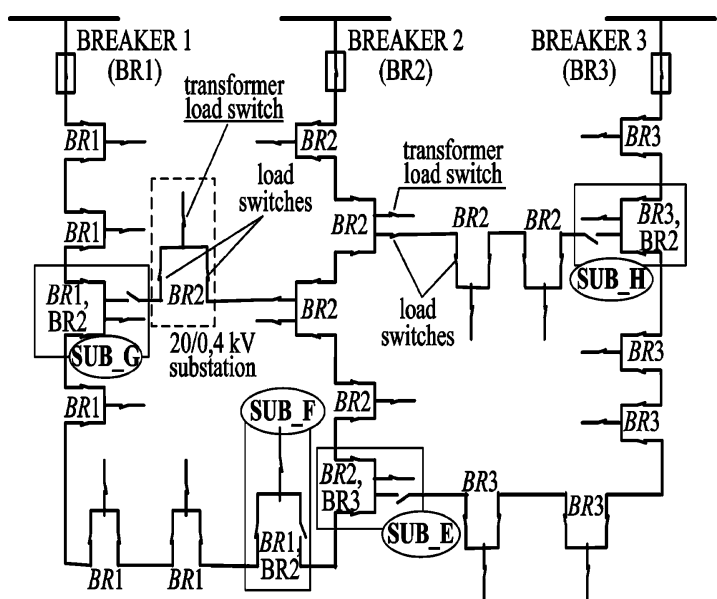

Fig. 3. MASCs of SUB_E, SUB_F, SUB_G, and SUB_H have gathered information for more than one breaker, according to Rules 1 and 2.

obey Expert's commands. Agent Expert initiates the fault isolation state and as soon as the fault has been isolated, it passes the control to Agents Com and Inter who cooperate to begin and complete the restoration state. Internally, the agents represent states with behavior classes that come into suspend or active mode according to specific messages coming from the agent's environment.

1) Steady State: On steady state, the Terminal Agents send messages with information about the state, the load, and the remaining loading capacities of the breakers to the adjacent MASCs. The corresponding information is propagated from one substation to the other according to the following rules:

Rule 1: An MASC does not send a message to an MASC that belongs to a substation located at the side of an open switch

Rule 2: An MASC does not send any message coming from an MASC that belongs to a substation, located at the side of an open switch.

The above rules block the looped circulation of the informative message. The implemented way of message propagation allows the MASCs which belong to substations that interconnect alternative power sources, to acquire information about the alternative power sources. Fig. 3 depicts a part of a typical underground distribution network. The symbolism " $B R 1, \mathrm{BR} 2$ " means that the corresponding MASC possesses data for more than one power source, while the notation in italics indicates the supplying source (for example the above MASC is aware of the loading condition of BREAKER 1 and BREAKER 2, while the supplying source of the MASCs substation is BREAKER 1). The tags attached to substations (SUB_F, SUB_G, etc.) are the identifiers of the corresponding substations.

Each MASC monitors three line voltages, three line currents, and the power of each local load switch. The procedure of detecting, locating, and isolating a fault has been implemented according to the following sequence of actual events: "fault commencing, raise of current—drop of voltage, breaker sensing, breaker tripping, loss of voltage, loss of current." The quantification of these events with respect to time is not deterministic. The time response of the breaker depends on the type of fault, the distance from the fault point, etc. The MASCs will not

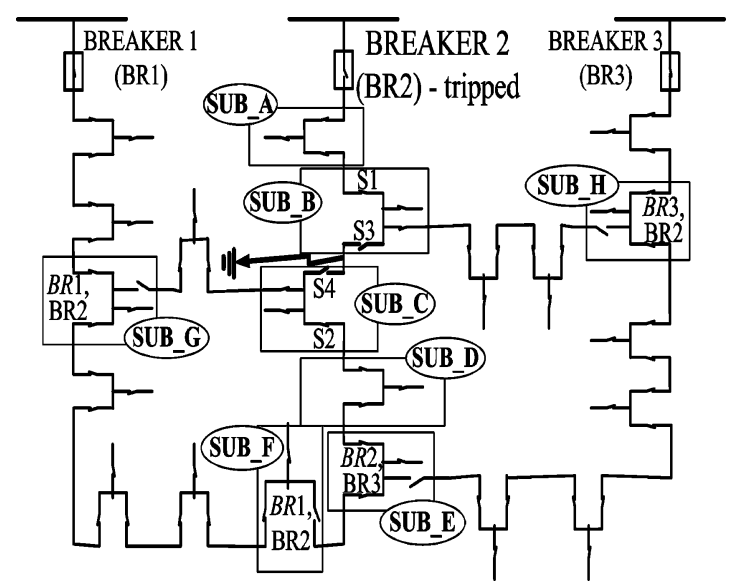

Fig. 4. Previous part of the grid with a fault detected and isolated between load switches S3 and S4.

change to fault isolation state unless they realize fault detection followed by total loss of voltage and total loss of current on all phases (fault cleared) or just total loss of voltage and current on all phases.

Agents Expert are the only ones responsible for altering the lists (1) of prior knowledge on steady state.

2) Fault Isolation State: As soon as the circuit breakers (CBs) have tripped to clear the fault, the MASCs will change to fault isolation state. Initially, MASCs of adjacent substations exchange messages containing their corresponding fault detection status according to the following constraint.

Constraint 1: An MASC will not send a positive detection message [i.e., fault(1)] to an MASC of a substation located at the side of an open switch.

Each MASC then compares:

- fault detection status of its own substation;

- fault detection status of the neighbored substations.

As a result, the MASCs of substations adjacent to the fault (Fig. 4, MASCs of SUB_B and SUB_C) will soon realize that a fault occurred between them, because the fault detection status will change from fault(1) to fault( 0 ) between those MASCs.

Usually a fault does not destroy the physical continuity of the cable conductor, given that both cable conductor size and cable joints/terminals have been rated according to the appropriate short-circuit ratings [18]. Nevertheless, the MASCs are capable of locating the fault, even in the case of conductor discontinuity, as shown in Subsection IV-D.

Thereafter, the MASCs proceed with fault isolation by opening the load switches located at the side of the fault (Fig. 4, MASCs of SUB_B and SUB_C open switches S3 and S4, respectively). Similar to steady state, Agents Expert are the only ones responsible for altering the lists (1) of prior knowledge.

3) Restoration State: The restoration procedure may then be initiated by the MASCs that isolated the fault. Each MASC is able to deduce the initially supplying breaker from its prior knowledge. The MASC located at the side of the initially supplying breaker (Fig. 4, MASC of SUB_B) will send a message to the MASC that is located at the side of a closed switch (Fig. 4, MASC of SUB_A) with the following content:

Message 1 : "fault_detect(1), breaker $(D), \operatorname{status}(1)$ " 
where $D$ is the identifier of the initially supplying breaker (Fig. 4, BREAKER 2).

Message 1 will be propagated and finally reach the corresponding Terminal Agent. The element "status(1)" is a command addressing the Terminal Agent to close the breaker and safely restore power to SUB_A and SUB_B. Along the message route, the MASCs enter restoration state successively; they exit restoration state as soon as they sense voltage restored.

On the other hand, the MASC that has not seen the fault but isolated it (Fig. 4, MASC of SUB_C), commences the following restoration procedure (the notation is the same as in Fig. 4).

MASC of SUB_C dynamically constructs a new list, which is called "Restoration Request List", of the form

$$
\begin{aligned}
& \text { Restoration RequestList : } \\
& \text { [breaker }(D, F), \\
& \quad \text { sub_req(SUB_C, } N U M, P, S T, S, N) \ldots]
\end{aligned}
$$

where value $D$ of element breaker() is the identifier of the breaker that tripped to clear the fault (Fig. 4, D is BR2), while $F$ is the remaining load that breaker with identifier $D$ can serve. $F$ is set to zero for BR2 in order to indicate that the corresponding breaker is left out of the restoration procedure.

The element sub_req() is dynamically added to the list and refers to the substation with the following attributes:

$N U M$ is a natural number that represents the dynamic position of the substation with respect to the fault $(N U M=1$ for SUB_C). $P$ is the prefault load of the substation. $S T$ is the state of switch S2 (Fig. 4, $S T=1$ ), $S$ is the prefault load of switch $\mathrm{S} 2, N$ is the number of switches of the substation $(N=4$ for SUB_C).

MASC of SUB_C then sends list (2) to SUB_D. MASC of SUB_D immediately enters the restoration state as a response to the received list, adds a new element sub_req() with its personal attributes to the list, and sends the new list to SUB_E. It should be stated that an MASC deducts the position with respect to the fault of the substation it belongs to by adding a unit to attribute $N U M$ of the element sub_req() that refers to the sender of the list.

On restoration state, the MASCs propagate the generated list (2) according to the following rules.

Rule 3: An MASC does not send the list to an MASC of a substation located at the side of an open switch.

Rule 4: An MASC does not send the list that comes from an MASC of a substation located at the side of an open switch.

Generally, the list's propagation stops at a MASC which possesses information for more than one power source (Fig. 4, MASCs of SUB_E, SUB_F, SUB_G, and SUB_H).

In the special case where the list (2) reaches a substation with more than 2 neighbors (i.e., a grid junction), the corresponding MASC proceeds to the following actions.

1) The MASC sends the list (according to Rules 3 and 4) toward one of the alternative directions and waits for a response.

2) On the arrival of the first response, the MASC sends the list (according to Rules 3 and 4) toward the other direction and waits for a second response.
A response is actually the "Restoration Request List" (2) returned from an MASC that owns information for an alternative power source. The updated list, which is included in the response, contains the element breaker $(D, F)$ that corresponds to the alternative power source. The decision upon the restoration strategy is made by the MASCs of the substations at the grid's junctions.

\section{Restoration Algorithms}

Two restoration algorithms are proposed.

Algorithm A: The MASC of the substation at the last grid's junction (Fig. 4, SUB_E) decides first in the row upon the restoration strategy. The decision concerns switching actions that have to be performed by the substations of the list (2). The switching actions refer to the switches that are included in the elements sub_req() of the above list.

The decision is made according to the following rule.

Rule 5a: An MASC decides which group of substations included in list (2) will remain out of power, starting from the substation with $N U M=1$ and proceeding successively until the remaining capacity of the alternative power source minus a safety margin is covered. The decision is formed in a "Command List"

$$
\begin{aligned}
& \text { Command List : } \\
& {[\text { breaker }(D, F) \text {, sub_req(SUB_C, } 1, P, 1, S, N) \text {, }} \\
& \text { SUB_D, } 2, P, 0, S, N)]
\end{aligned}
$$

which is actually the "Restoration Request List" (2), containing the switching actions of the substations that belong to the main part of the line (Fig. 4, SUB_C and SUB_D). A switching action of a substation which corresponds to element sub_req(), is represented in the "Command List" by means of changing value $S T$ of element sub_req() to zero (i.e., off). The "Command List" also contains the element breaker $(D, F)$ that corresponds to the alternative power source, where $F$ is the new remaining capacity of the selected breaker. The "Command List" is then sent to the direction of the substation with a $N U M$ value less than the local one.

The next MASC in the row bases its decision upon the switching actions included in the "Command List."

- If a switching action refers to a substation with $N U M$ greater than or equal to the local one, the MASC implements Rule 5a, without the part of the line isolated by the substation that corresponds to the switching action.

- If a switching action refers to a substation with $N U M$ less than the local one, then the MASC may safely open the local load switch (if necessary) and implement Rule $5 \mathrm{a}$, without the part of the line isolated by the local switching action.

Algorithm B: The MASC of the substation at the first grid's junction (Fig. 4, SUB_C) decides first in the row upon the restoration strategy. Algorithm B requires that the "Restoration Request List" (2) is returned complete and unedited to the above MASC. The decision is made according to the following rule. 
Rule 5b: An MASC decides which group of substations included in list (2) will remain out of power starting from the substation with the larger $N U M$ value and proceeding successively until the remaining capacity of the alternative power source minus a safety margin is covered.

The decision is formed in the same way as in Algorithm A. A similar "Command List" (3) is then sent to the direction of the substation with an NUM value greater than the local one.

The next MASC in the row bases its decision upon the switching actions included in the "Command List."

- If a switching action refers to a substation with an $N U M$ value less than the local one, the MASC implements Rule 5b, without the part of the line isolated by the substation that corresponds to the switching action.

- If a switching action refers to a substation with an $N U M$ value greater than or equal to the local one, then the MASC may safely open (if necessary) the load switch that isolates the part of the lineup to the local substation and implement Rule $5 \mathrm{~b}$ without the isolated part.

In both algorithms, the main concern of the MASCs that belong to substations at grid junctions is to avoid the engagement of two sources in parallel. The reasoning of the MASCs is a responsibility of Agents Com which creates the dynamic lists(2), (3) and propagates them along the line. Agents Inter take care of the switching actions and update the lists of prior knowledge, as soon as a switching action has taken place.

Conclusions regarding the implementation of both algorithms are included in Section VI.

\section{Agent Communications}

Two types of communications occur during execution time, namely intracommunity and intercommunity, respectively. The communication procedures among the agents of the same community fall into the first category. They are specified by the FIPA standards and represent interactions of independent control threads occupied by agents living in the same environment.

The intercommunity communications concern the corresponding procedures that take place between neighbored substations and among the substations and the control centers. Simple agent communication language (ACL) messages are being exchanged among JADE agent-platforms while the necessary actions are performed mainly by Agents Com.

The MV line serves as the physical communication channel. For the purpose of the present research, the amount of data is not a decisive issue and, in principle, the CENELEC A-band (3-95 kHz available for utilities; European standard EN50065) allows a sufficient data-transfer rate. The choice of utilizing the medium-voltage (MV) line for the communication needs results in several advantages regarding the autonomy of the system. The distribution network will rely on its own resources, while dependencies on extra telecommunication paths and networks will be avoided. The behavior of an MV cable with respect to coupling schemes for MV PLC is presented in [19]. In this work, the communications are mainly established between adjacent 20/0.4 distribution substations, where the length of the MV cable is less than $1 \mathrm{~km}$. Therefore, communication signals travel short distances along the cable conductors and more important: signal attenuation is low.

The proposed system has been designed to allow the exploitation of the networking potentials provided by the distribution grid. On the other hand, special care has been taken in order to keep modulation frequencies at low levels and thus to achieve decent signal propagation. Moreover, the amount of exchanged data is kept low. As previously stated, communications are established between two successive 20/0.4-kV substations. In this context, communications breakdowns have been considered as issues of bad communications between two successive substations.

On steady state, the data exchange concerns the propagation of an informative message containing information about the supplying breaker condition. A communication problem will cause an undelivered message exception on the side of the sender MASC. The latter will report the situation backwards, to the supplying source direction.

On the fault isolation state, the data exchange concerns the state of fault detectors of adjacent substations. Communication problems caused by the consequences of a short circuit on the cable conductor will trigger an undelivered message exception on both sides of the occurred fault because both MASCs will try to send an informative message containing the state of their fault detectors to each other. Thereafter, both MASCs will deduce that the fault occurred between them and proceed with fault isolation.

With the power restoration state, the data exchange concerns the propagation of the dynamic lists. Communication problems will cause an undelivered message exception on the side of the sender MASC.

- If the communication problem occurs during the propagation of the "Restoration Request List," the sender MASC's actions will depend on the Algorithms implemented:

- In Algorithm A, the sender MASC will open the local load switch and return the list as a "Command List" containing the local switching action.

- In Algorithm B, the "Restoration Request List" follows a complete route from the first, with respect to the fault's position, grid's junction to the last, and back to the first one. If the communication problem occurs in the first part of the list's route, then the sender MASC will return the list unedited. The list will be finalized at the sender's MASC position. If the communication problem occurs in the second part of the list's route, then the sender MASC will act as in Algorithm A.

- If the communication problem occurs during the propagation of the "Command List," then the sender MASC will act according to the contents of the list as follows.

- If the list contains a switching action that precedes the sender's location, then the sender will return the command list without any other action.

- If the list contains a switching action that follows the sender's location, the sender will then open the local 


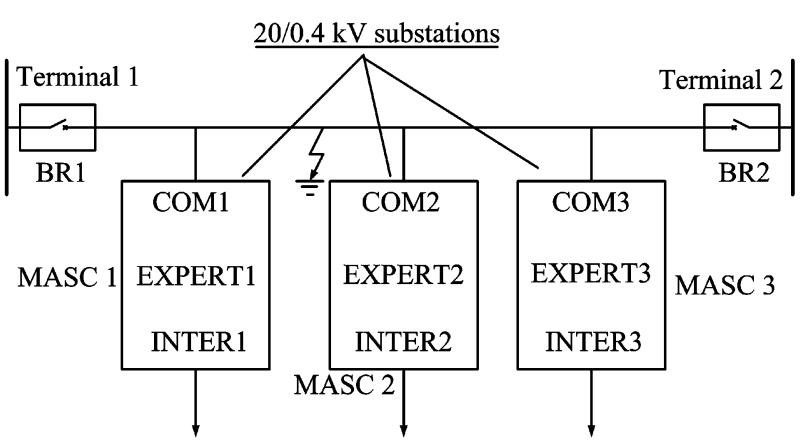

Fig. 5. First case of study: an MV cable with three distribution substations.

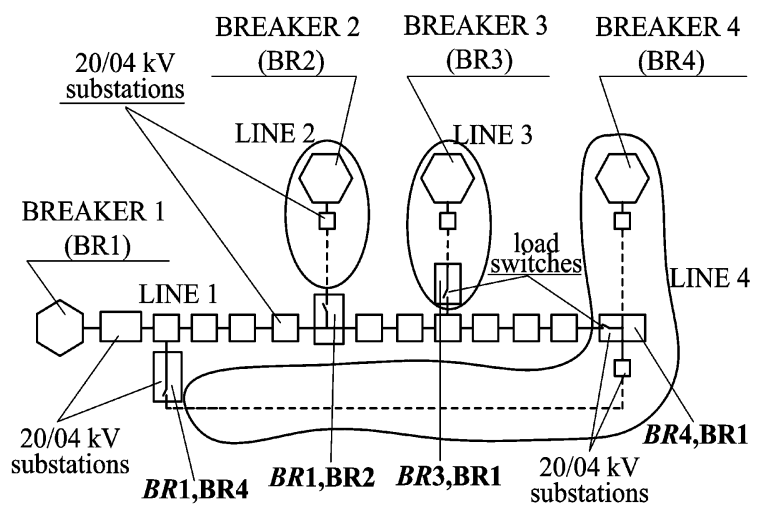

Fig. 6. Second case of study: distribution network with four interconnected lines.

load switch, update the command list with the new switching action, and return the list as a confirmation message concerning the switching action that took place.

\section{CASES STUdied}

Two cases were considered in order to examine the operation of the proposed MAS.

The first case aimed at evaluating the abilities of the MASCs to work unsynchronized and manage intranet and serial communication ports. The simple topology of Fig. 5 was selected for the purposes of the first case.

Initially, BR1 was assumed to supply the line. A fault between MASC 1 and MASC 2 is considered. BR2 is the only alternative power source for the line. BR2 is assumed with enough remaining capacity, to supply the entire line. Results and comments regarding the simulation of the simple topology of Fig. 5, are included in Section VI.

The second case aimed at the evaluation of the restoration algorithms. The topology of Fig. 6 was selected. Fig. 6 illustrates a real part of the underground distribution network of Thessaloniki, Greece. It consists of $420-\mathrm{kV}$ distribution lines (LINE $\# 1, \# 2, \# 3$, \#4) which supply 13, 9, 10, and 11 20/0.4-kV distribution substations, respectively.

The second case was focused on LINE 1 which is the circuit supplied by BREAKER 1. The initial load of LINE 1 was the actual load of 5.4 MVA, as reported on Friday 13/02/04 from 19:15 till 19:45. LINE \#2, \#3, and \#4 serve as the alternative power sources for the loads of LINE 1. LINE 1 was assumed

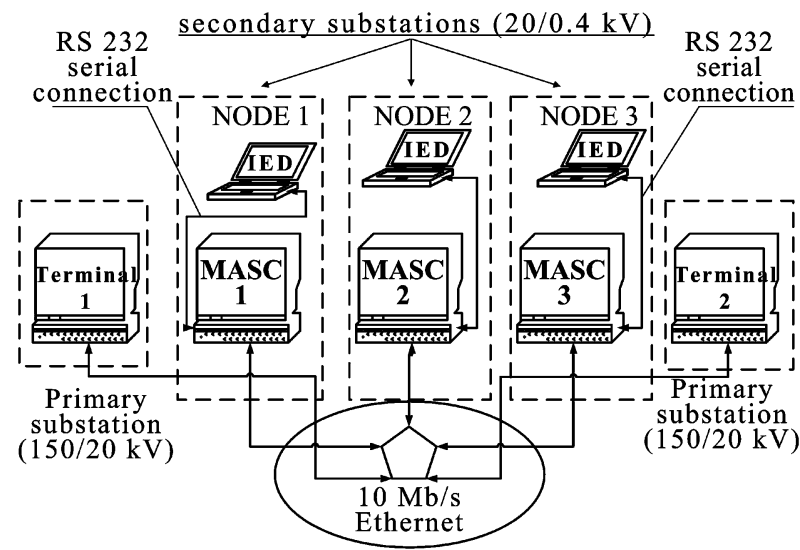

Fig. 7. First case of study: simulation topology.

uniformly loaded (each substation supplied approximately 415 $\mathrm{kVA}$ ). Several scenarios concerning the remaining capacity of the alternative power sources were examined. Results and comments regarding the simulation of the topology of Fig. 6 are included in Section VI.

\section{Simulations}

A software prototype of the proposed MAS was developed to simulate the first Case of Study. The simulation topology is illustrated in Fig. 7.

Each PC was accommodating a JADE platform with a threeagent community. Two additional PCs, corresponding to the $150 / 20-\mathrm{kV}$ primary substations, were also connected to the same network. (Fig. 7). Operational inputs were provided by input data files conforming to the COMTRADE [20] standard. Additional software objects simulating the IEDs were installed on laptops connected to the PCs via RS-232 serial ports.

Regarding MASC 1, the fault condition was simulated with a line of the input data files containing a fault detection value, followed by a group of lines containing fault detection along with zero values for line current and voltages. Regarding MASC 2 and MASC 3, the fault condition was simulated by a group of lines containing zero values for line currents and voltages. The above form of the input files was selected in order to simulate nonsynchronized MASCs operation. The assumption that all of the MASCs are aware of the alternative power source (Fig. 5, BR2) was made. The following results were acquired according to the time stamps of the messages appearing on the PCs' screens

12:05:15 Terminal 1: sending message to Com1

$$
\text { BR1: } 1
$$

remain: 1500

12:05:16 Com1: received message from Terminal 1

$$
\text { BR1: } 1
$$

remain: 1500

12:05:16 Com1: sending message to Expert 1

$$
\text { BR1: } 1
$$

remain: 1500

12:05:16 Com1: sending message to Com2

BR1: 1 remain: 1500 
12:05:16 Expert1: received message from Com1

BR1: 1

remain: 1500

12:05:17 Expert1: query data from Inter1

12:05:17 Inter1: sending data to Expert1

12:05:17 Com2: received message from Com1

BR1: $1 \quad$ remain: 1500

12:05:17 Com2: sending message to Expert 2

BR1: $1 \quad$ remain: 1500

12:05:17 Com2: sending message to Com3

BR1: 1

remain: 1500

12:05:17 Expert2: received message from Com2

BR1: 1

remain: 1500

12:05:18 Expert2: query data from Inter2

12:05:18 Inter2: sending data to Expert2

12:05:18 Com3: received message from Com2

BR1: $1 \quad$ remain: 1500

12:05:18 Com3: sending message to Expert3

BR1: $1 \quad$ remain: 1500

12:05:18 Expert3: received message from Com3

BR1: 1

remain: 1500

12:05:19 Expert3: query data from Inter3

12:05:19 Inter3: sending data to Expert3

12:09:23 Expert1: sending message to Com1

fault: 1

12:09:23 Com1: message received from Expert1

fault: 1

12:09:24 Com1: sending message to Com2 fault: 1

12:09:26 Expert2: sending message to Com2 fault: 0

12:09:26 Com2: received message from Expert2 fault: 0

12:09:27 Com2: sending message to Com1 fault: 0

12:09:28 Com2: received message from Com1 fault: 1

12:09:28 Com1: received message from Com2 fault: 0

12:09:28 Com2: sending message to Expert2

Com1 fault: 1

12:09:29 Com1: sending message to Expert1

Com2 fault: 0

12:09:29 Expert2: sending message to Inter2

left switch: 0

12:09:29 Expert1: sending message to Inter1

right switch: 0

12:09:30 Inter1: sending message to Expert1

right switch: $0 \mathrm{ok}$

12:09:30 Inter2: sending message to Expert2

left switch: 0 ok

12:09:31 Expert1: sending message to Com1

BR1: 1

12:09:31 Expert2: sending message to Com2

BR2: 1

12:09:32 Com1: sending message to Terminal1

BR1: 1

12:09:32 Terminal1: received message from Com1

BR1: 1

12:09:32 Com2: sending message to Com3

BR2: 1

12:09:34 Terminal2: received message from Com3:

BR2: 1.

The first line of each message shows the action which takes place at a specific time, while the second line shows the content of the exchanged messages. Some messages have been omitted due to a lack of space.

The results indicate that the agents exhibited the desirable behavior regarding the synchronization of the system Although MASC 1 and MASC 2 realized fault conditions on different time spots, they resolved the case and proceeded with fault isolation and power restoration without any operating conflicts or behavioral exceptions.

The corresponding expected times in the CENELEC A-band depend on the bandwidth provided by the PLC solutions available in the market, as well as on the maximum expected message size. Regarding narrowband PLC, an average transmission speed up to $4.8 \mathrm{~kb} / \mathrm{s}$ is usually considered [21]. In the case of FIPA-ACL bit-efficient encoding [22], the size of the ACL 


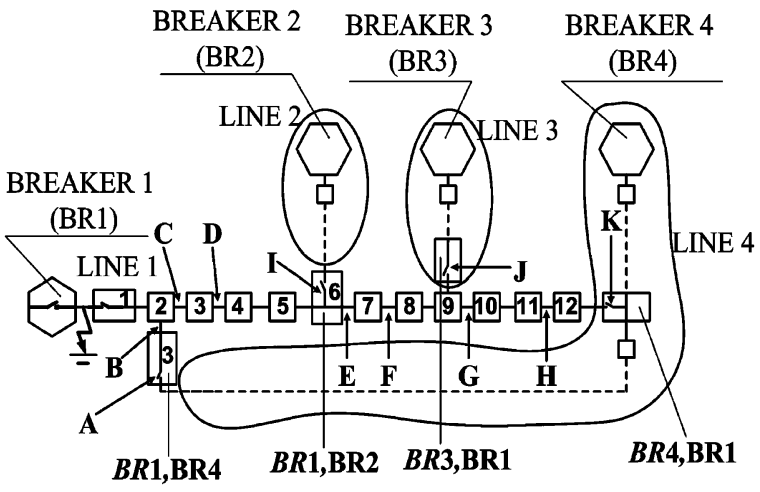

Fig. 8. Test case 1 topology.

TABLE I

Results of Test CASE 1, IN Relation to Fig. 8

\begin{tabular}{|c|c|c|c|c|}
\cline { 2 - 5 } \multicolumn{1}{c|}{} & Messages & $\begin{array}{c}\text { Circuit } \\
\text { open } \\
\text { at: }\end{array}$ & $\begin{array}{c}\text { Circuit } \\
\text { closed } \\
\text { at: }\end{array}$ & $\begin{array}{c}\text { Power } \\
\text { Restored } \\
{[\mathrm{kVA}]}\end{array}$ \\
\hline $\begin{array}{c}\text { Algorithm } \\
\text { A }\end{array}$ & 40 & $\begin{array}{c}\text { B, C, } \\
\text { E, G }\end{array}$ & $\begin{array}{c}\text { A, I, J, } \\
\text { K }\end{array}$ & 4565 \\
\hline $\begin{array}{c}\text { Algorithm } \\
\text { B }\end{array}$ & 43 & $\begin{array}{c}\text { D, F, } \\
\text { H }\end{array}$ & A, I, J & 4980 \\
\hline
\end{tabular}

messages is relatively small [23] and by all means, less than the available bandwidth. Therefore, agent transactions duration time, which in a $10 / 100-\mathrm{Mb} / \mathrm{s}$ network is less than a second, is not expected to exceed 1 or $2 \mathrm{~s}$. Results acquired from the implementation of the IIOP Message Transport Protocol [24] with a connection of $9600 \mathrm{~b} / \mathrm{s}$ also verify the expected times mentioned above [25].

The second case of study was simulated according to the following.

Technical constraints as well as available resources postulated the apportionment of the substations MASCs to six PCs. IED's objects were embodied in the JADE container of each MASC along with the data input files. The PCs were connected via a $10 / 100-\mathrm{Mb} / \mathrm{s}$ Ethernet. It must be noted that the absolute performance of the system with respect to time has been decreased, since each PC accommodates 7-8 MASCs. Thus, the simulation of the second case of study indicates a relative performance of the restoration algorithms in terms of amount of exchanged messages (among MASCs) and restoration efficiency. The results were acquired from the inspection of the lists of prior knowledge, after the finalization of the restoration procedure. The description and results of three indicative test cases are presented in the following:

Test case 1 (Fig. 8): Fault at the beginning of LINE 1. The remaining capacity of BREAKERS 2, 3, 4 is the one third of LINE 1 load (approximately $1800 \mathrm{kVA}$ ). Numbers 1, 2, $3, \ldots, 12$ attached to the substations indicate the ordering of the corresponding substations with respect to the fault's position. Table I summarizes the results of test case 1.

Generally, Algorithm B requires a larger amount of exchanged messages than Algorithm A. Nevertheless, Algorithm B performed better than Algorithm A, in Test case

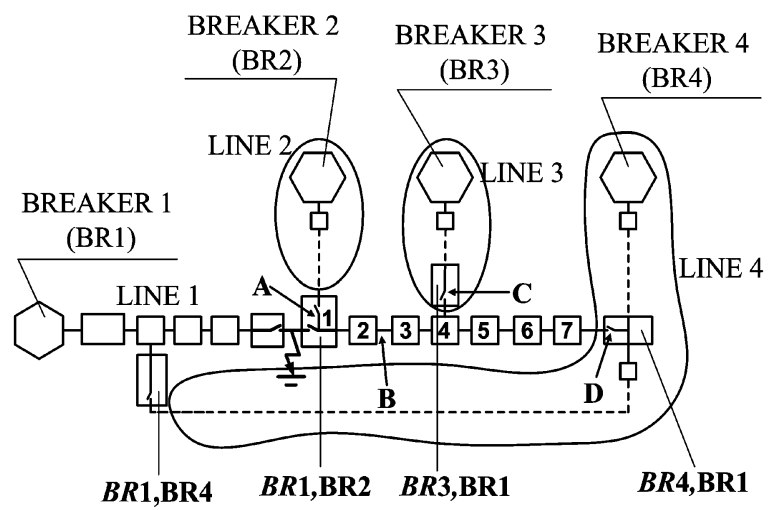

Fig. 9. Test case 2 topology.

1, with respect to restoration efficiency. The difference between the two algorithms lies in the way they manage the part of the line that has not detected the fault.

Algorithm A prioritizes the power sources controlled by the remotest, with respect to the fault, grid junctions. Therefore, Algorithm A performs better than Algorithm $\mathrm{B}$ in the cases where those power sources have larger remaining capacities than the ones controlled by grid junctions closer to the fault. On the other hand, Algorithm $\mathrm{B}$ prioritizes the power sources controlled by the grid junctions which are close to the fault. Therefore, Algorithm B performs better than Algorithm A in the case where those power sources have larger remaining capacities than the ones controlled by remote grid junctions.

The above conclusions are best illustrated in the following test cases.

Test case 2 (Fig. 9): The position of the fault is shown in Fig. 9. The test case focuses on the part of the line that has not detected the fault. The other part of the line will be restored as soon as the fault is isolated. Numbers $1,2, \ldots, 7$ attached to the substations indicate the ordering of the corresponding substations with respect to the fault's position. The remaining capacity of BREAKER 2 is approximately $968 \mathrm{kVA}$. BREAKERS 3 and 4 have zero remaining capacity. Table II summarizes the results of test case 2 .

Algorithm B performed better than algorithm A because the former prioritized BREAKER 2 which is also the alternative power source with the highest remaining capacity. The larger amount of exchanged messages in Algorithm $B$ is justified by the switching action confirmation which MASC of substation \#2 had to send back to MASC of substation \#1.

Test case 3 (Fig. 10): The position of the fault is the same as in test case 2 . Numbers $1,2, \ldots, 7$ attached to the substations indicate the ordering of the corresponding substations with respect to the fault's position. The remaining capacity of BREAKER 4 is approximately $968 \mathrm{kVA}$. BREAKERS 2 and 3 have zero remaining capacity. Table III summarizes the results of test case 3 .

Algorithm A performed better than algorithm B because the former prioritized BREAKER 4 which is also the alternative power source with the highest remaining capacity. The larger 
TABLE II

RESUlts of Test CASE 2, IN RELATION TO Fig. 9

\begin{tabular}{|c|c|c|c|c|}
\cline { 2 - 5 } \multicolumn{1}{c|}{} & Messages & $\begin{array}{c}\text { Circuit } \\
\text { open } \\
\text { at: }\end{array}$ & $\begin{array}{c}\text { Circuit } \\
\text { closed } \\
\text { at: }\end{array}$ & $\begin{array}{c}\text { Power } \\
\text { Restored } \\
{[\mathrm{kVA}]}\end{array}$ \\
\hline $\begin{array}{c}\text { Algorithm } \\
\text { A }\end{array}$ & 16 & $\begin{array}{c}\text { A, C, } \\
\text { D }\end{array}$ & - & 0 \\
\hline $\begin{array}{c}\text { Algorithm } \\
\text { B }\end{array}$ & 20 & $\begin{array}{c}\text { B, C, } \\
\text { D }\end{array}$ & A & 830 \\
\hline
\end{tabular}

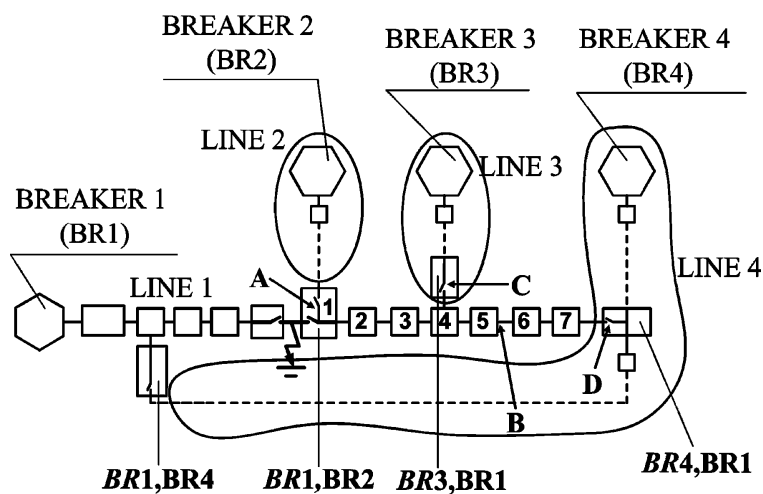

Fig. 10. Test case 3 topology.

TABLE III

Results of Test CASe 3, in Relation to Fig. 10

\begin{tabular}{|c|c|c|c|c|}
\cline { 2 - 5 } \multicolumn{1}{c|}{} & Messages & $\begin{array}{c}\text { Circuit } \\
\text { open } \\
\text { at: }\end{array}$ & $\begin{array}{c}\text { Circuit } \\
\text { closed } \\
\text { at: }\end{array}$ & $\begin{array}{c}\text { Power } \\
\text { Restored } \\
{[\mathrm{kVA}]}\end{array}$ \\
\hline $\begin{array}{c}\text { Algorithm } \\
\text { A }\end{array}$ & 18 & $\begin{array}{c}\text { A, C, } \\
\text { B }\end{array}$ & D & 830 \\
\hline $\begin{array}{c}\text { Algorithm } \\
\text { B }\end{array}$ & 16 & $\begin{array}{c}\text { B, C, } \\
\text { D }\end{array}$ & - & 0 \\
\hline
\end{tabular}

amount of exchanged messages in Algorithm A is justified by the switching action confirmation which the MASC of substation \#5 had to send back to the MASC of the substation that controls BREAKER 4.

\section{CONCLUSION}

The perspectives of the proposed MAS architecture are quite promising. The implementation of the MAS as presented in this work will result in a desirable autonomy, in terms of self-management, of the power distribution network. The entire network will become a living organism with self-healing capabilities. The power restoration procedures will become less time consuming. The electrical network will not be forced to sustain successive short circuits, as the traditional "trial and error" methodologies will be obviated. As a result, the diminishing cost of fault management will probably compensate the system's installation initial investment.

Moreover, from the agent-technology aspect, the authors presented a concept of designing adaptive MAS which are application oriented; the system does not implement certain learning procedures regarding the network management, since the experience provided by the domain experts is quite enough to enable the agents to locate occurring faults and restore power to healthy areas. However, adaptive behavior is necessary since

- the system ought not to become unstable when a network topological alternation takes place due to the activities of the agent communities;

- the system must maintain stability even if the actions of the agent communities are not synchronized;

- the operation of the system must remain safe and must not oppose human intervention.

\section{REFERENCES}

[1] C.-C. Liu, S. J. Lee, and S. S. Venkata, "An expert system operational aid for restoration and loss reduction of distribution systems," IEEE Trans. Power Syst., vol. 3, no. 2, pp. 619-626, May 1988.

[2] Y.-Y. Hsu, F.-C. Lu, Y. Chien, J. P. Liu, J. T. Lin, P. H. S. Yu, and R. R. T. Kuo, "An expert system for locating distribution system faults," IEEE Trans. Power Del., vol. 6, no. 1, pp. 366-372, Jan. 1991.

[3] H.-J. Lee and Y.-M. Park, "A restoration aid expert system for distribution substations," IEEE Trans. Power Del., vol. 11, no. 4, pp. 1765-1770, Oct. 1996

[4] T. Sakaguchi and K. Matsumoto, "Development of a knowledge based system for power system restoration," IEEE Trans. Power App. Syst., vol. PAS-102, no. 2, pp. 320-329, Feb. 1983.

[5] D. S. Kirschen and T. L. Volkmann, "Guiding a power system restoration with an expert system," IEEE Trans. Power Syst., vol. 6, no. 2, pp. 558-566, May 1991.

[6] M. M. Adibi, "New approaches in power system restoration," IEEE Trans. Power Syst., vol. 7, no. 4, pp. 1428-1434, Nov. 1992.

[7] M. Nordman, M. Lehtonen, J. Takala, E. Suvinen, M. Hyvarinen, and E. Antila, "TETRA radio in monitoring and control of secondary substations," in Proc. Inst. Elect. Eng., Apr. 9-12, 2001, pp. 283-286.

[8] M. Amin, "Toward self-healing energy infrastructure systems," IEEE Comput. Appl. Power, vol. 14, no. 1, pp. 20-28, Jan. 2001.

[9] T. Nagata and H. Sasaki, "A multi-agent approach to power system restoration,” IEEE Trans. Power Syst., vol. 17, no. 2, pp. 457-462, May 2002.

[10] M. Nordman and M. Lehtonen, "An agent concept for managing electrical distribution networks," IEEE Trans. Power Del., vol. 20, no. 2, pp. 696-703, Apr. 2005.

[11] J. Ferber, Multi-Agent Systems-An Introduction to Distributed Artificial Intelligence. Reading, MA: Addison-Wesley, 1999, pp. 30-35.

[12] T. Short, Electric Power Distribution Handbook. Boca Raton, FL: CRC, 2004, pp. 441-445.

[13] IntelliTEAM II, IntelliTEAM II, Automatic Restoration System S\&C Electric Company [Online]. Available: http://www.sandc.com.

[14] Power Distribution Network Guide Public Power Corporation S.A., Greece, 1987.

[15] JADE—Java Agent Development Framework (C) 2000 TILab S.p.A., (C) 2001 TILab S.p.A., (C) 2002 TILab S.p.A., (C) 2003 TILab S.p.A. [Online]. Available: http://jade.tilab.com.

[16] FIPA: The Foundation for Intelligent Physical Agents, [Online]. Available: http://www.fipa.org., FIPA standards.

[17] Amzi Prolog + Logic Server, @ Amzi! Inc., 1994-2002 [Online]. Available: http://www.amzi.com.

[18] E. W. G. Bungay and D. McAllister, Electric Cables Handbook, 2nd ed. London, U.K.: Blackwell, 1990, pp. 141-158, 168-171.

[19] P. A. A. F. Wouters, P. C. J. M. vanderWielen, J. Veen, P. Wagenaars, and E. F. Steennis, "Effect of cable load impedance on coupling schemes for MV power line communication," IEEE Trans. Power Del., vol. 20 , no. 2, pt. 1 , pp. 638-645, Apr. 2005.

[20] Standard Common Format for Transient Data Exchange (COMTRADE) for Power Systems, IEEE Std. C37.111-1999, Revision of IEEE Std. C37.111-1991, Oct. 1999.

[21] CE-0740 Modem for Data Transmission via Medium Voltage Distribution Networks [Online]. Available: http://www.sat-automation.com/ pdf/ce074xe.pdf.

[22] FIPA ACL Message Representation in Bit-Efficient Encoding Specification, FIPA Spec. SC00069G FIPA Spec. SC00069G, Dec. 2002 [Online]. Available: http://www.fipa.org/specs/fipa00069/index.html.

[23] F. Zambonelli and M. Klusch, Cooperative Information Agents V. Berlin, Germany: Springer-Verlag, 2001, pp. 260-262. 
[24] FIPA Agent Message Transport Protocol for IIOP Specification, FIPA Spec. SC00075G FIPA Spec. SC00075G, Dec. 2002 [Online]. Available: http://www.fipa.org/specs/fipa00075/index.html.

[25] R. Unland, M. Calisti, and M. Klusch, Software Agent-Based Applications, Platforms and Development Kits. Basel, Switzerland: Birkhäuser Verlag, 2005, pp. 311-314.

Ioannis S. Baxevanos was born in Naoussa, Greece, on October 21, 1974. He received the Dipl.-Eng. degree from the Department of Electrical and Computer Engineering, Aristotle University of Thessaloniki, Thessaloniki, Greece, in 2001 .

Since 2001, he has been with the Power Systems Laboratory, Department of Electrical and Computer Engineering, Aristotle University of Thessaloniki. His research activities concern the implementation of artificial intelligence technology in the power systems field. His special interests are power distribution systems analysis, control and protection, with special emphasis on power distribution systems automation.
Dimitris P. Labridis (S'88-M'90-SM'00) was born in Thessaloniki, Greece, on July 26, 1958. He received the Dipl.-Eng. and the Ph.D. degrees from the Department of Electrical and Computer Engineering, Aristotle University of Thessaloniki, in 1981 and 1989, respectively.

During 1982-2000, he was a Research Assistant, Lecturer, and then Assistant Professor with the Department of Electrical and Computer Engineering, Aristotle University of Thessaloniki. Since 2001, he has been with the Department of Electrical and Computer Engineering, Aristotle University of Thessaloniki, as an Associate Professor. His special interests are power system analysis with special emphasis on the simulation of transmission and distribution systems, electromagnetic and thermal field analysis, artificial intelligence applications in power systems, power-line communications, and distributed energy resources. 